radius $1 / n$ having restrictions at $h_{\nu}$. Let $F$ denote the family of all collections $G_{(v, n)}$. It can easily be shown that $F$ is the required family. Axiom 2 is evidently satisfied.

Note. If in space $T^{\alpha}$ the point $P$ be said to be the sequential limit point of a type $\omega_{\alpha}$ sequence of points $\left[P_{i}\right]_{i}{ }^{\omega_{\alpha}}$ if and only if it is true that if $R$ is a region containing $P$, then $R$ contains a residue of sequence $\left[P_{i}\right]_{i} \omega_{\alpha}$, there then exist type $\omega_{\alpha}$ convergent sequences of points. Thus we have been led by a series of apparently natural definitions to the existence of uncountable convergent sequences of points in certain spaces of uncountably many dimensions.

Austin, Tex.

\title{
NOTE ON THE LOCATION OF ZEROS OF THE DERIVATIVE OF A RATIONAL FUNCTION WHOSE ZEROS AND POLES ARE SYMMETRIC IN A CIRCLE*
}

\section{J. L. WALSH}

1. Introduction. The most general function which effects a 1-to-m conformal transformation of the interior of the unit circle $|z|=1$ onto itself is of the form

$$
r(z)=\lambda \prod_{k=1}^{m} \frac{z-\alpha_{k}}{1-\bar{\alpha}_{k} z}, \quad\left|\alpha_{k}\right|<1,|\lambda|=1 ;
$$

so the location of the zeros of the derivative $r^{\prime}(z)$ is of considerable interest. The zeros and poles of $r(z)$ are symmetric in the unit circle. Moreover a typical transcendental function bounded in the unit circle is the Blaschke product (assumed convergent)

$$
B(z)=\prod_{k=1}^{\infty} \frac{\bar{\alpha}_{k}}{\left|\alpha_{k}\right|} \frac{z-\alpha_{k}}{\bar{\alpha}_{k} z-1},
$$

which is the limit for $|z|<1$ of a sequence of functions each of form (1). It is of some significance in studying the behavior of $B(z)$ to know exactly or approximately the zeros of $B^{\prime}(z)$. The object of the present note is to give some fairly simple but elegant results on the derivatives of both $r(z)$ and $B(z)$. Application is made also to the critical points of certain harmonic functions.

2. Derivative of a rational function. We first obtain the following result:

\footnotetext{
* Presented to the Society, December 30, 1938.
} 
Theorem 1. Let a circle $K$ lie interior to $C:|z|=1$ and contain in its interior all the points $\alpha_{1}, \alpha_{2}, \cdots, \alpha_{m}$. Then $K$ contains in its interior precisely $m-1$ zeros of $r^{\prime}(z)$, where $r(z)$ is defined by (1). The inverse with respect to $C$ of the interior of $K$ contains all the remaining finite zeros of $r^{\prime}(z)$.

The zeros $\alpha_{k}$ of $r(z)$ and the poles $1 / \bar{\alpha}_{k}$ of $r(z)$ are mutually inverse in $C$, so the poles of $r(z)$ lie exterior to $K$, and the conclusion follows from the results due to Bôcher and the present writer.*

If the points $\alpha_{1}, \alpha_{2}, \cdots, \alpha_{m}$ are given, Theorem 1 enables us, by the use of a number of different circles $K$, to obtain a region interior to $C$ containing in its interior both all the $\alpha_{k}$ and all the $m-1$ zeros of $r(z)$ interior to $C$. Still another result is somewhat more specific:

THEOREM 2. If a circular region $G$ bounded by a circle $\Gamma$ orthogonal to $C:|z|=1$ contains in its interior no point $\alpha_{k}$, then the region $G$ contains in its interior no finite zero of $r^{\prime}(z)$, where $r(z)$ is defined by (1).

In Theorem 2 (and in similar situations below) such a circle as $\Gamma$ may be a straight line. In proving the theorem it will be a convenience to have for reference the following lemma, whose proof is immediate:

LEMma 1. In the field of force due to $n$ unit particles $Q_{1}, Q_{2}, \cdots, Q_{n}$ each repelling with a force equal to the inverse distance, the force at a point $P$ may be found as follows. Let $Q_{k}^{\prime}$ be the inverse of the point $Q_{k}$ in the unit circle whose center is $P$. Let $Q^{\prime}$ be the center of gravity of the points $Q_{1}^{\prime}, Q_{2}^{\prime}, \cdots, Q_{n}^{\prime}$. Then the force at $P$ due to the $n$ particles $Q_{k}$ is $n$ times the vector $\overline{Q^{\prime}} \bar{P}$.

The finite zeros of $r^{\prime}(z)$ not multiple zeros of $r(z)$ are the positions of equilibrium in the field of force due to equal repelling particles each of mass +1 situated at each of the points $\alpha_{k}$, and equal repelling particles each of mass -1 (that is, attracting particles of mass +1 ) situ-

* Bôcher (Proceedings of the American Academy of Arts and Sciences, vol. 40 (1906), pp. 469-484) proves: If two circular regions $T_{1}$ and $T_{2}$ have no point in common and contain respectively the roots of two binary forms $f_{1}$ of degree $p_{1}$ and $f_{2}$ of degree $p_{2}$, then $T_{1}$ and $T_{2}$ contain respectively $p_{1}-1$ and $p_{2}-1$ roots of the jacobian of $f_{1}$ and $f_{2}$. A circular region is here and below a closed region of the extended plane bounded by a circle or line.

Bôcher's result was applied by Walsh (Transactions of this Society, vol. 19 (1918), pp. 291-298) to the study of the derivatives of a rational function: If two circular regions $T_{1}$ and $T_{2}$ have no point in common and contain respectively all the zeros and all the poles of a rational function $f(z)$ of degree $n$, then all of the zeros of the derivative $f^{\prime}(z)$ lie in $T_{1}$ and $T_{2}$ except that there are two additional zeros of $f^{\prime}(z)$ at infinity if $f(z)$ has no pole there. Except for these two possible zeros, there are precisely $n-1$ zeros of $f^{\prime}(z)$ in $T_{1}$, and if $f(z)$ has no multiple poles, there are precisely $n-1$ zeros of $f^{\prime}(z)$ in $T_{2}$. 
ated at each of the points $1 / \bar{\alpha}_{k}$; the law of force is that of the inverse distance.* Under the hypothesis of Theorem 2 , let the point $P$ lie in the region $G$. The inverses of $C$ and $\Gamma$ in the unit circle whose center is $P$ are circles $C^{\prime}$ and $\Gamma^{\prime}$, which are orthogonal to each other; $\Gamma^{\prime}$ is necessarily a proper circle; the circle $C^{\prime}$ separates the images $Q_{k}^{\prime}$ of the $\alpha_{k}$ from the images $Q_{k}^{\prime \prime}$ of the $1 / \bar{\alpha}_{k}$; the points $Q_{k}^{\prime}$ and $Q_{k}^{\prime \prime}$ lie in the image of the complement of $G$, namely the interior of $\Gamma^{\prime}$; the point $P$ may or may not lie interior to $\Gamma^{\prime}$. For convenience in exposition, let us orient the plane so that both $C^{\prime}$ and $\Gamma^{\prime}$ are symmetric in a vertical line (that is to say, so that the two points of intersection of $C^{\prime}$ and $\Gamma^{\prime}$ lie on a horizontal line), with each point $Q_{k}^{\prime}$ above the corresponding point $Q_{k}^{\prime \prime}$; of course $Q_{k}^{\prime}$ and $Q_{k}^{\prime \prime}$ are mutually inverse in $C^{\prime}$. Then the center of gravity $Q^{\prime}$ of the points $Q_{k}^{\prime}$ lies above the center of gravity of the points $Q_{k}^{\prime \prime}$. Consequently, (Lemma 1 ) the resultant of the attractive forces is not equal and opposite to the resultant of the repelling forces; the point $P$ cannot be a position of equilibrium in the field of force; the point $P$ cannot be a multiple zero of $r(z)$, hence cannot be a zero of $r^{\prime}(z)$; so Theorem 2 is established.

An immediate consequence of Theorem 2 is the following corollary:

COROLlaRY. Under the hypothesis of Theorem 2, if all the points $\alpha_{k}$ lie on $\Gamma$, then all the finite roots of $r^{\prime}(z)$ also lie on $\Gamma$.

In any given situation, there are a number of circles $\Gamma$ of the kind required in Theorem 2 that can be drawn to delimit a region in which the zeros of $r^{\prime}(z)$ lie, so we have the theorem:

TheOREM 3. Let the points $\alpha_{k}$ be given interior to $C:|z|=1$, and let $\Pi$ be the smallest (closed) curvilinear polygon interior to $C$ bounded by arcs of circles $\Gamma$ orthogonal to $C$, such that $\Pi$ contains each of the points $\alpha_{k}$, and where each $\Gamma$ bounds a circular region containing in its interior no $\alpha_{k}$. Then $\Pi$ contains on or within it all the zeros of $r^{\prime}(z)$ interior to $C$, where $r(z)$ is given by (1).

Theorem 3 is the precise non-euclidean analogue of the classical theorem of Lucas that all the zeros of the derivative $p^{\prime}(z)$ of a polynomial $p(z)$ lie in the smallest convex polygon containing all the roots of $p(z)$. For the function $w=r(z)$ maps $|z|<1$ conformally in a 1 -to- $m$ manner onto $|w|<1$, and is the most general function defining such a map, just as $w=p(z)$ maps $|z|<\infty$ onto $|w|<\infty$ conformally in a 1-to- $m$ manner, and is the most general function defining such a map. The derivatives of both $r(z)$ and $p(z)$ vanish precisely $m-1$

\footnotetext{
* See Bôcher, loc. cit., Walsh, loc. cit.
} 
times in the regions involved. The regions specified in Theorem 3 and Lucas's theorem are both the smallest convex sets containing the roots of the given function, in the respective senses of non-euclidean and euclidean geometry. The two theorems are respectively invariant under arbitrary one-to-one conformal transformation onto itself of the non-euclidean plane $|z|<1$ and of the euclidean plane $|z|$ $<\infty$.

An arbitrary simply connected region $R$ of the w-plane with more than one boundary point can be mapped conformally onto $|z|<1$; a function $f(w)$ analytic in $R$, whose modulus is continuous in the corresponding closed region and constant on the boundary but not in $R$, corresponds under the map to a constant multiple of a function $r(z)$ as defined by (1). Thus Theorem 3 yields the result that if non-euclidean geometry is defined in $R$ by means of the conformal map onto $|z|<1$, then the zeros of $f^{\prime}(w)$ in $R$ lie in the smallest non-euclidean convex polygon containing the zeros of $f(w)$ in $R$; it is a theorem due to Denjoy that the number of zeros of $f^{\prime}(w)$ in $R$ is one less than the number of zeros of $f(w)$ in $R$. Theorems 5-10 may similarly be generalized at once by a conformal map.

If fixed points $\alpha_{k}$ interior to $|z|=r$ are given, the most general function analytic in $|z|<r$, vanishing precisely in the points $\alpha_{k}$, whose modulus is continuous in $|z| \leqq r$ and equal to $r^{n}$ on $|z|=r$, is given by

$$
f(z) \equiv \lambda \prod_{k=1}^{n} \frac{z-\alpha_{k}}{1-\bar{\alpha}_{k} z / r^{2}}, \quad|\lambda|=1 ;
$$

equation (1) is the special case $r=1$. When $r$ becomes infinite, $f(z)$ approaches the function $p(z)=\lambda \prod_{k=1}^{n}\left(z-\alpha_{k}\right)$; the derivative $f^{\prime}(z)$ approaches $p^{\prime}(z)$; the zeros of $f^{\prime}(z)$ in $|z|<r$ approach the zeros of $p^{\prime}(z)$; a variable circle through two fixed points $z$ independent of $r$ and orthogonal to $|z|=r$ has a radius which becomes infinite with $r$, for the center of such a variable circle lies exterior to $|z|=r$. Thus Lucas's theorem is a limiting case of Theorem 3.

3. Both zeros and poles interior to $C$. Lucas's theorem admits of an extension to rational functions:*

* Compare the theorems of Bôcher and Walsh already quoted; also M. B. Porter, Proceedings of the National Academy of Sciences, vol. 2 (1916), pp. 247-248; Walsh, Transactions of this Society, vol. 24 (1922), pp. 31-69; Marden, Transactions of this Society, vol. 32 (1930), pp. 658-668. The theorems of Bôcher and Walsh previously quoted are concerned with all the zeros and poles of the functions of the hypothesis, whereas Theorem 4 involves explicitly only the finite zeros and poles. But the former results contain the essential content of Theorem 4 . 
THEOREM 4. Let $r(z)$ be a rational function of $z$ with the finite zeros $\alpha_{1}, \alpha_{2}, \cdots, \alpha_{m}$ and the finite poles $\beta_{1}, \beta_{2}, \cdots, \beta_{n}$. If $L$ is a line separating each $\alpha_{j}$ from each $\beta_{k}$, then no finite zero of $r^{\prime}(z)$ lies on L. Consequently, if such lines $L$ exist, all the finite zeros of $r^{\prime}(z)$ lie in two unbounded convex point sets which are separated by every $L$ and which contain respectively the points $\alpha_{j}$ and the points $\beta_{k}$.

If all the points $\alpha_{j}$ and all the points $\beta_{k}$ lie on a line, and if no $\alpha_{j}$ separates a pair of the $\beta_{k}$ and no $\beta_{k}$ separates a pair of the $\alpha_{j}$, then all finite zeros of $r^{\prime}(z)$ lie on that line, and lie on the smallest infinite segments containing respectively all the $\alpha_{j}$ and none of the $\beta_{k}$, and all of the $\beta_{k}$ and none of the $\alpha_{j}$.

Theorem 4 has a precise analogue in the non-euclidean case:

THEOREM 5. Let $r(z)$ be a rational function of $z$ with the zeros $\alpha_{1}, \alpha_{2}, \cdots, \alpha_{m}$ and poles $\beta_{1}, \beta_{2}, \cdots, \beta_{n}$ interior to $C:|z|=1$, and the poles $1 / \bar{\alpha}_{k}$ and zeros $1 / \bar{\beta}_{k}$ exterior to $C$, and having no other zeros or poles. If $L$ is a circle orthogonal to $C$, and if $L$ separates each $\alpha_{j}$ from each $\beta_{k}$, then no zero of $r^{\prime}(z)$ lies on $L$ interior to $C$. Consequently, if a circle $L$ exists, all the zeros of $r^{\prime}(z)$ interior to $C$ lie in two closed point sets $\Pi_{1}$ and $\Pi_{2}$ which are bounded by circles orthogonal to $C$ each of which separates all the $\alpha_{j}$ not lying on it from all the $\beta_{k}$ not lying on it; these two point sets $\Pi_{1}$ and $\Pi_{2}$ are separated by every $L$; they contain, respectively, all the $\alpha_{j}$ and all the $\beta_{k}$.

If all the points $\alpha_{j}$ and all the points $\beta_{k}$ lie on a circle $K$ orthogonal to $C$, and if no $\alpha_{j}$ lies on an arc of $K$ interior to $C$ bounded by a pair of the $\beta_{k}$, and if no $\beta_{k}$ lies on an arc of $K$ interior to $C$ bounded by a pair of the $\alpha_{j}$, then all the zeros of $r^{\prime}(z)$ interior to $C$ lie on $K$, and lie on the two arcs of $K$ bounded by $C$ which are the shortest arcs of $K$ interior to $C$ terminated at one end by $C$ and containing respectively all the points $\alpha_{j}$ and all the points $\beta_{k}$.

The function $r(z)$ of Theorem 5 is the quotient of two arbitrary functions of the kind defined by (1).

We shall establish Theorem 5 by means of the easily proved lemma:

Lemma 2. Let $L$ be a circle orthogonal to $C:|z|=1$, and let a particle of mass +1 be situated at $\alpha$ interior to $C$ but not on $L$, and a particle of mass -1 be situated at $1 / \bar{\alpha}$. Then the corresponding force at a point $P$ of $L$ interior to $C$ has a nonvanishing component orthogonal to $L$ in the sense directed from the side of $C$ on which $\alpha$ lies.

Lemma 2 may be proved at once by inversion in the unit circle whose center is $P$. Under this inversion let $C, L, \alpha, 1 / \bar{\alpha}$ correspond, 
respectively, to $C^{\prime}, L^{\prime}, \alpha^{\prime}, \beta^{\prime}$, so that $L^{\prime}$ is a line passing through the center of the (proper) circle $C^{\prime}$; the points $\alpha^{\prime}$ and $\beta^{\prime}$ are mutually inverse with respect to $C^{\prime}$ and are both separated or both not separated from $P$ by $L^{\prime}$, unless $L$ is a straight line and consequently $P$ lies on $L^{\prime}$. But $\alpha^{\prime}$ lies exterior to $C^{\prime}$ and $\beta^{\prime}$ interior to $C^{\prime}$. The force at $P$ due to the two given particles is represented by the sum of the vectors $\alpha^{\prime} P$ and $P \beta^{\prime}$; that is to say, is represented by the vector $\alpha^{\prime} \beta^{\prime}$. The vector $\alpha^{\prime} \beta^{\prime}$ has a nonvanishing component orthogonal to $L^{\prime}$, in the sense of the perpendicular from $\alpha^{\prime}$ onto $L^{\prime}$. This is equivalent to the statement of the lemma.

The proof of Theorem 5 is now immediate. Let $L$ be orthogonal to $C$ and separate each $\alpha_{j}$ from each $\beta_{k}$. Consider the field of force whose positions of equilibrium determine the zeros of $r^{\prime}(z)$. Then the force at a point $P$ of $L$ interior to $C$ due to the particles $\alpha_{j}$ and $1 / \bar{\alpha}_{j}$ has a nonvanishing component in the sense directed from the side of $L$ on which $\alpha_{j}$ lies. The force at $P$ due to the particles $\beta_{k}$ and $1 / \bar{\beta}_{k}$ has a nonvanishing component in this same sense, so $P$ cannot be a position of equilibrium. But $P$ cannot be a multiple zero of $r(z)$, and hence cannot be a zero of $r^{\prime}(z)$. Theorem 5 is established.

Theorem 4 can be proved from Theorem 5 by a limiting process.

Theorem 5 essentially includes Theorem 2 , where the points $\beta_{k}$ of Theorem 5 do not exist. Theorem 2 may also be established from Lemma 2. Indeed, both lemmas as well as Theorems 2 and 5 can be proved from the fact that in the field of force due to two particles of masses equal in magnitude but opposite in sign, the lines of force are precisely the circles through those particles. The sense of the force at every point of such a circle is directed from the repelling particle toward the attracting particle.

It follows that in the last part of Theorem 5 an arc of $K$ interior to $C$ bounded by two $\alpha_{k}$ (or by two $\beta_{k}$ ) and containing in its interior no $\alpha_{k}$ or $\beta_{k}$ contains in its interior at least (as a matter of fact, precisely) one zero of $r^{\prime}(z)$. A similar remark applies to the Corollary to Theorem 2, to the latter part of Theorem 6 , and under suitable conditions to Theorem 8 .

Under the conditions of the last part of Theorem 5, it may occur that zeros of $r^{\prime}(z)$ lie on $C$ but not on $K$, as we now show by means of an example. Let $K$ be an arbitrary proper circle orthogonal to $C$, and let $M$ denote the diameter of $K$ through $O: z=0$. Let $\alpha_{1}$ be chosen on $K$ interior to $C$ but not on $M$, let $\beta_{1}$ be the inverse of $\alpha_{1}$ in $M$, which is also on $K$, and choose $m=n=1$. Considerations of symmetry and the fact that the lines of force due to the particles at $\alpha_{1}$ and $1 / \bar{\alpha}_{1}$ are circles through those points, show that the two intersections of $M$ 
with $C$ are zeros of $r^{\prime}(z)$; but these intersections do not lie on the circle $K$.

4. Derivative of a Blaschke product. The Blaschke product (assumed convergent) defined by (2) is the limit for $|z|<1$ of the sequence

$$
B_{n}(z)=\prod_{k=1}^{k=n} \frac{\bar{\alpha}_{k}}{\left|\alpha_{k}\right|} \frac{z-\alpha_{k}}{\bar{\alpha}_{k} z-1},
$$

and $B_{n}(z)$ is of type (1). Convergence of $B_{n}(z)$ to $B(z)$ is uniform for $|z| \leqq r<1$, and convergence of the sequence $B_{n}^{\prime}(z)$ to the function $B^{\prime}(z)$ is also uniform in the closed region $|z| \leqq r<1$. If a point $z_{0}$ with $\left|z_{0}\right|<1$ is not a limit point of zeros of the functions $B_{n}^{\prime}(z)$, it follows by a well known theorem due to Hurwitz that $z_{0}$ cannot be a zero of $B^{\prime}(z)$. Consequently, we have from Theorem 2 the following theorem:*

THEOREM 6. If a circular region $G$ bounded by a circle $\Gamma$ orthogonal to $C:|z|=1$ contains in its interior no point $\alpha_{k}$, then the region $G$ contains in its interior no zero of $B^{\prime}(z)$ in $|z|<1$, where $B(z)$ is defined by (2).

In particular, if all the points $\alpha_{k}$ lie on $\Gamma$, so also do all the zeros of $B^{\prime}(z)$ in $|z|<1$.

A somewhat simpler but less precise result than Theorem 6 is the next theorem:

THEOREM 7. Let the points $\alpha_{k}$ lie interior to a circle $C_{1}$ which is tangent to $C:|z|=1$ internally. Then all the finite zeros of $B^{\prime}(z)$ interior to Clie in $C_{1}$.

For each $n$, all of the points $\alpha_{1}, \alpha_{2}, \cdots, \alpha_{n}$ lie in a circle interior to but concentric with $C_{1}$; so by Theorem 1 the zeros of the derivatives of the corresponding partial product interior to $C$ lie in the interior of this smaller circle. Theorem 7 thus follows from Hurwitz's theorem.

A similar method of proof yields an analogue of Theorem 5:

TheOREM 8. Let $B(z)$ be the Blaschke product (2), and let

$$
D(z)=\prod_{k=1}^{\infty} \frac{\bar{\beta}_{k}}{\left|\beta_{k}\right|} \frac{z-\beta_{k}}{\bar{\beta}_{k} z-1}, \quad \quad\left|\beta_{k}\right|<1,
$$

* It is to be remarked in connection with Theorem 6 that an arbitrary function $f(z)$ analytic and bounded for $|z|<1$, which has boundary values of constant modulus for normal approach to $|z|=1$, is a constant multiple of a Blaschke product of type (2). 
also be a convergent Blaschke product. If $L$ is a circle orthogonal to $C:|z|=1$, and if $L$ separates each $\alpha_{j}$ from each $\beta_{k}$, then no zero of $r^{\prime}(z)$, where $r(z) \equiv B(z) / D(z)$, lies on $L$ interior to $C$. Consequently, if such $a$ circle $L$ exists, all the zeros of $r^{\prime}(z)$ interior to $C$ lie in two closed point sets $\Pi_{1}$ and $\Pi_{2}$ which are bounded by circles orthogonal to $C$ each of which separates all the $\alpha_{j}$ not lying on it from all the $\beta_{k}$ not lying on it; these two point sets $\Pi_{1}$ and $\Pi_{2}$ are separated by every $L$; they contain respectively all the $\alpha_{j}$ and all the $\beta_{k}$.

In Theorem 3 we have emphasized the location of the zeros of $r^{\prime}(z)$ interior to $C$. This is no actual restriction, for each result applies effectively also to the zeros of $r^{\prime}(z)$ even exterior to $C$. For instance if we set $r_{1}(z) \equiv 1 / r(1 / z)$, where $r(z)$ is represented by (1), we have

$$
r_{1}(z) \equiv \frac{1}{\lambda} \prod_{k=1}^{m} \frac{z-\bar{\alpha}_{k}}{1-\alpha_{k} z}, \quad\left|\bar{\alpha}_{k}\right|<1,
$$

so that Theorem 3, for example, applies to the function $r_{1}(z)$, and yields results on the zeros of $r^{\prime}(z)$ exterior to $C$. In fact, all finite zeros of $r^{\prime}(z)$ exterior to $C$ lie in the inverse with respect to $C$ of the polygon $\Pi$ of Theorem 3. But if no $\alpha_{k}$ vanishes, the derivative $r^{\prime}(z)$ vanishes at infinity whether or not the point at infinity lies in the inverse in $C$ of the polygon $\Pi$.

A remark similar to the one just made applies not only to Theorem 3 but also to Theorems 5, 6, 7, and 8. But the present results do not treat directly the zeros of $r^{\prime}(z)$ on $C$ in Theorems 5 and 8 .

A linear transformation of the complex variable which transforms $C:|z|=1$ into the axis of reals yields for Theorems 1-3, 5-8 analogous results on the derivatives of functions of the forms

$$
\prod_{k=1}^{m} \frac{z-\alpha_{k}}{z-\bar{\alpha}_{k}}, \quad \prod_{k=1}^{\infty} \frac{z-\alpha_{k}}{z-\bar{\alpha}_{k}} .
$$

5. Critical points of harmonic functions. By methods that the pressent writer has developed elsewhere, ${ }^{*}$ the new results just established can be extended to apply to the critical points of harmonic functions. The detailed proof, which we leave to the reader, may be based directly on the theorems already proved.

Theorem 9. In the extended plane let $C$ be a circle, let the Jordan curves $C_{1}^{\prime}, C_{2}^{\prime}, \cdots, C_{\nu}^{\prime}$ not intersect $C$, all lie on the same side of $C$, and be respectively symmetric to the Jordan curves $C_{1}^{\prime \prime}, C_{2}^{\prime \prime}, \ldots, C_{\nu}^{\prime \prime}$ with

\footnotetext{
* Proceedings of the National Academy of Sciences, vol. 20 (1934), pp. 551-554.
} 
respect to $C$. Let no two of the curves $C_{j}^{\prime}$, and no two of the curves $C_{k}^{\prime \prime}$ intersect, and let the totality of those curves constitute the complete boundary of a region $R$. Let the function $u(x, y)$ be harmonic in the interior of $R$ and continuous in the corresponding closed region, and let $u(x, y)$ take on the value unity on every $C_{k}^{\prime}$ and the value minus unity on every $C_{k}^{\prime \prime}$.

(1) If $G$ is a region bounded by a circle $\Gamma$ orthogonal to $C$, and if $G$ contains in its interior no point exterior to $R$, then $G$ contains in its interior no critical point of $u(x, y)$.

(2) If a circle $K^{\prime}$ separates all the curves $C_{k}^{\prime}$ from $C$, then $K^{\prime}$ separates precisely $\nu-1$ critical points of $u(x, y)$ from $C$; the inverse $K^{\prime \prime}$ of $K^{\prime}$ in $C$ separates precisely $\nu-1$ critical points of $u(x, y)$ from $C$.

Theorem 10. In the extended plane let $C$ be a circle, let the Jordan curves $C_{1}^{\prime}, C_{2}^{\prime}, \cdots, C_{\mu}^{\prime}, K_{1}^{\prime}, K_{2}^{\prime}, \cdots, K_{\nu}^{\prime}$ not intersect $C$, all lie on the same side of $C$, and be respectively symmetric to the Jordan curves $C_{1}^{\prime \prime}, C_{2}^{\prime \prime}, \cdots, C_{\mu}^{\prime \prime}, K_{1}^{\prime \prime}, K_{2}^{\prime \prime}{ }^{\prime \prime} \cdots, K_{\nu}^{\prime \prime}$ with respect to $C$. Let no two of the curves $C_{1}^{\prime}, \cdots, C_{\mu}^{\prime}, K_{1}^{\prime}, \cdots, K_{\nu}^{\prime}$ intersect, and let the totality of the curves $C_{j}^{\prime}, K_{j}^{\prime}, C_{j}^{\prime \prime}, K_{j}^{\prime \prime}$ constitute the complete boundary of a region $R$. Let the function $u(x, y)$ be harmonic in the interior of $R$ and continuous in the corresponding closed region, and let $u(x, y)$ take on the value unity on every $C_{j}^{\prime}$ and $K_{j}^{\prime \prime}$ and the value minus unity on every $C_{j}^{\prime \prime}$ and $K_{j}^{\prime}$.

If $L$ is a circle orthogonal to $C$, and if $L$ separates each $C_{j}^{\prime}$ from each $K_{k}^{\prime}$, then no critical point of $u(x, y)$ lies on $L$ but not on $C$. Consequently, if such a circle $L$ exists, the critical points of $u(x, y)$ not on $C$ lie in two regions $\Pi_{1}$ and $\Pi_{2}$ which are bounded by circles $L^{\prime}$ orthogonal to $C$ each of which separates (except for points of intersection with $L^{\prime}$ ) each $C_{j}^{\prime}$ from each $K_{k}^{\prime}$, and each $C_{j}^{\prime \prime}$ from each $K_{k}^{\prime \prime}$. The regions $\Pi_{1}$ and $\Pi_{2}$ are separated by every $L$; they contain, respectively, all the $C_{j}^{\prime}$ and $C_{j}^{\prime \prime}$, and all the $K_{j}^{\prime}$ and $K_{j}^{\prime \prime}$.

In Theorem 10 we may make the convention that the case $\nu=0$ is not excluded, and that the corresponding restriction on $L$ is that $L$ shall intersect no $C_{j}^{\prime}$ and shall separate no two of the circles $C_{j}^{\prime}$. If this convention is made, Theorem 10 includes the essence of Theorems $1,2,3,4,5,9$, and by a limiting process may be used to prove also Theorems 6,7 , and 8 .

Theorems 9 and 10 extend to certain situations in which the boundary of the region $R$ has a finite or infinite number of components which are not necessarily Jordan curves.

HARVARD UNIVERSITY 